\title{
Complementing the European earth observation and geographic information body of knowledge with a business-oriented perspective
}

\author{
Barbara Hofer $^{1}$ (D) | Sven Casteleyn ${ }^{2}$ | Estefanía Aguilar-Moreno ${ }^{2}$ | \\ Eva-Maria Missoni-Steinbacher $^{1}$ | Florian Albrecht ${ }^{1}$ | Rob Lemmens ${ }^{3}$ | \\ Stefan Lang $^{1}$ | Jochen Albrecht ${ }^{4}$ | Martyna Stelmaszczuk-Górska ${ }^{5}$ | \\ Glenn Vancauwenberghe $^{6}$ | Aida Monfort-Muriach ${ }^{2}$
}

${ }^{1}$ Interfaculty Department of Geoinformatics Z_GIS, University of Salzburg, Salzburg, Austria

${ }^{2}$ GEOTEC, Institute of New Imaging Technologies, Universitat Jaume I, Castelló de la Plana, Spain

${ }^{3}$ Geo-Information Processing (ITC), University of Twente, Enschede, the Netherlands

${ }^{4}$ Department of Geography, Hunter College CUNY, New York, NY, USA

${ }^{5}$ Department for Earth Observation, Friedrich Schiller University Jena, Jena, Germany

${ }^{6}$ Spatial Applications Division, KU Leuven, Leuven, Belgium

\section{Correspondence}

Barbara Hofer, Interfaculty Department of Geoinformatics-Z_GIS, University of Salzburg, Salzburg, Austria.

Email: Barbara.Hofer@sbg.ac.at

\section{Funding information}

Education, Audiovisual and Culture Executive Agency, Grant/Award Number: Erasmus+ Sector Skill Alliance for the EO4GEO proj; Ramón y Cajal Programme of the Spanish government, Grant/Award Number: Sven Casteleyn is funded by the Ramón y Cajal Pr

\begin{abstract}
A body of knowledge (BoK) is an inventory of knowledge or concepts of a domain that serves as a reference vocabulary for various purposes, such as the development of curricula, the preparation of job descriptions, and the description of occupational profiles. To fulfill its purpose, a BoK needs to be up-to-date and ideally widely accepted by academia as well as the private and public sectors. This article presents the initiative taken in the Earth observation and geographic information (EO* $\left.{ }^{*} \mathrm{I}\right)$ domain to provide a current, comprehensive education- and business-oriented EO* GI BoK called EO4GEO BoK. In particular, an approach to strengthen the business-oriented perspective in the EO4GEO BoK is presented. This approach is based on the analysis of professional tasks and the mapping of these tasks to concepts and skills contained in the BoK. A critical reflection of the proposed approach that is based on the experiences gained during a workshop complements this article.
\end{abstract}




\section{1 | INTRODUCTION}

The Earth observation (EO) and geographic information (GI) domain, referred to as the EO*GI domain in this article, is a high-skill sector that is continuously affected by technological advances, with increasing amounts of data available and a strong need to foster user uptake to draw value from these data and take advantage of the huge investments being made. As a key element in exploiting these technological advances and data, clearly delineating and defining the fundamental concepts of this rapidly expanding and evolving field of study is critical. A body of knowledge (BoK), defined as "a comprehensive inventory of the intellectual content that defines a field" (DiBiase et al., 2007), has been shown to be an adequate solution in various fields [e.g., Abran, Moore, Bourque, Dupuis, and Tripp (2004) for software engineering; Morris, Patel, and Wearne (2000) for project management; DiBiase et al. (2007) for geographic information science and technology].

In its simplest form, a body of knowledge consists of a controlled vocabulary (Mundie \& Ruefle, 2012), defining an agreed-upon terminology. In a more elaborate form, it formally describes the key concepts and relationships between them, along with their key attributes and associated terminology in a formal structure, such as an ontology (Ahearn et al., 2013). Such a BoK, as a well-defined, agreed-upon representation of the domain, may play a pivotal role in aligning educational offerings and professional demand (Wallentin, Hofer, \& Traun, 2015). From the educational perspective, the BoK can steer (model) curriculum design (Abran et al., 2004), from the basis for defining, annotating curricula and syllabi (Kedron, Frazier, Greene, \& Mitchell, 2016), textbooks (Frazier, Wikle, \& Kedron, 2017), or for accreditations of study programs and vocational trainings. From the perspective of the individual trainee, student, or expert aspiring to an additional qualification, the BoK shall offer guidance on choosing suitable courses or educational programs as well as an orientation for self-assessments, CV profiles, personal career plans, etc. From the professional perspective, the BoK can be used to facilitate professional certifications (DiBiase et al., 2007), for domain-specific applications, or to define or annotate occupational profiles or job offers (Gruijthuijsen, Pontes, Vancauwenberghe, Vandenbroucke, \& Olijslagers, 2019).

The EO4GEO BoK, developed as part of the Erasmus + Sector Skills Alliance project EO4GEO (http://eo4geo.eu), builds on a revision of the European geographic information science and technology (GIS\&T) BoK (Vandenbroucke \& Vancauwenberghe, 2016), which itself stems from the original UCGIS GIS\&T BoK (Wilson, 2014). Leveraging the input from a network of over 150 domain experts, the European GIS\&T BoK is currently being extended with EO concepts and their relationships, as well as related skills that indicate the practical use of these concepts. This revision process is performed using traditional techniques, such as expert interviews, discussion sessions, and workshops, and is organized in working groups that each cover a particular knowledge area in the field. While historically shown to be an adequate process, the consecutive versions of the BoK and the current revision process are mainly driven by academia, and the resulting BoK is therefore sometimes critiqued as being too theoretical, only applicable in the educational field, and too far from practical knowledge and applications required in the workplace.

To address this criticism, and to complement traditional means of BoK definition and revision, this article presents a novel approach aimed at better aligning the academically oriented EO4GEO BoK with the business, professional, and industrial perspective. This approach is based on the analysis of business processes, which are subsequently broken down into tasks routinely performed by the EO*GI workforce, along with the required skills and knowledge. Currently, a set of 30 business processes is available, collected from practitioners in the field, who are personally involved in the work processes, which lists over 200 tasks. The business process descriptions form the basis for identifying occupational profiles that are themselves linked to job offer descriptions and indicate which academic as well as vocational training programs need to be developed.

While occupational profiles may be quite diverse and depend on the actual professional setting, the particularities of the $\mathrm{EO}^{*} \mathrm{Gl}$ sector allow for a generalized view of such profiles, including the following: (a) core profiles requiring a solid understanding of the technical and scientific key concepts (e.g., to generate information products in a service provider company); (b) managerial profiles relying on a general understanding of the existence and 
dynamics of key concepts (e.g., to properly distribute tasks or define tender specifications); (c) complementary profiles with a basic understanding of the processes to perform technical or administrative support tasks; (d) peripheral or linked profiles of neighboring disciplines and domains (e.g., a town planner) with general knowledge about the technical and application-oriented advancements occurring in the sector. The process of detailing bespoke curricula and training programs requires that the tasks linked to occupational profiles are understood in detail. Detailing tasks means that the knowledge and skills required for executing tasks are documented such that the training material can be designed accordingly.

A notable contribution regarding the alignment of industry needs and educational programs is made by the U.S.-based National Geospatial Technology Center of Excellence (GeoTech Center, http://www.geotechcenter. org/). The GeoTech Center conducted workshop series using the developing a curriculum method (DACUM) for identifying key tasks in geospatial professions on which 2-year college curricula are based. Also, they currently analyze core competencies in geospatial education with the major aim of reducing the initial long list of core competencies to a manageable list for further use in curriculum design. The authors are, however, not aware of a direct integration of this work into a domain-specific BoK.

The approach presented in this article proposes a detailed description of tasks from business processes based on concepts and skills included in the EO4GEO BoK. Thereby, the BoK serves the purpose of a vocabulary that is aligned with requirements from the business perspective and then serves as a foundation for curriculum design. It is demonstrated that the mapping exercise serves to evaluate the current state of development of the EO4GEO BoK and complements it with additional concepts and especially skills.

In Section 2, we provide a historical perspective on the EO4GEO BoK and detail its design and supporting tools in Section 3. Section 4 exemplifies the methodology for decomposing business processes and Section 5 , mapping between tasks, skills, and concepts. Then, in Section 6, experiences of applying the methodology in practice are discussed. In Section 7, we conclude and explain how the approach contributes to the EO4GEO BoK.

\section{2 | THE EO4GEO BoK-HISTORICAL PERSPECTIVE}

The development and maintenance of a BoK is a collaborative endeavor that requires a representative network of practitioners and experts contributing content, a governance structure to regulate the knowledge collection and management processes (e.g., agreements on scope and structure of the content, revision and updating processes), and a technical solution supporting the governance process, including the publication of the BoK. Below, we briefly sketch the history of the EO4GEO BoK, before going into more detail on the people, processes, and tools in place for its creation.

The origin of the EO4GEO BoK is the original GIS\&T BoK, published in book form in 2006 (DiBiase et al., 2007). It was defined using a top-down approach, where a team of 7 editors and 70 contributors created the hierarchically organized BoK. The hierarchical structure was extended to form an ontological representation, and an elaborate computational platform called BoKWIKI was built to support BoK browsing, navigation, and collaborative updating (Ahearn et al., 2013). However, this ontologized BoK and the corresponding BoKWIKI were not implemented for widespread consumption in the U.S., and were not publicly available apart from a proof-of-concept demo. In its current form, the GIS\&T BoK follows a curated encyclopedic approach (see https://gistbok.ucgis. org/), where concepts, now called "topics," are made available in the form of a structured, citable article, that is continuously added to or improved.

Since the first GIS\&T BoK was published in 2006, awareness grew that a European perspective on this US-led initiative was needed (Toppen \& Reinhardt, 2009) due to EU-specific initiatives, policies, and regulations. (e.g., the Bologna process for higher education, the INSPIRE initiative). These ideas crystallized into the EU-funded Lifelong Learning project GI-N2K (http://www.gi-n2k.eu/the-project/), which forked the digitized version of the original US GIS\&T BoK, contained in the BoKWIKI, and used it as a starting point to create the first European version of 
the BoK (Vandenbroucke \& Vancauwenberghe, 2016; Wallentin et al., 2015). This updating process followed a bottom-up, participatory strategy, whereby stakeholders (e.g., academics, practitioners, experts) were able to suggest, discuss, and contribute content. In the context of the Erasmus + Sector Skills Alliance project EO4GEO (http://eo4geo.eu), the GI-N2K BoK was further elaborated and extended to include the Earth observation domain. Both the GI-N2K and the EO4GEO BoKs are organized according to an underlying ontological structure of concepts and relationships, and revisions were performed collaboratively by a network of European experts.

Remarkably, all the aforementioned BoK versions were created in academia-led initiatives: the US versions under the auspices of the University Consortium of Geographic Information Science (UCGIS), and the EU versions within EU projects predominantly consisting of academic partners.

\section{3 | THE EO4GEO BOK-DESIGN AND SUPPORTING TOOLS}

The EO4GEO BoK is based on an underlying ontological structure and consists of:

- Concepts, which correspond to pieces of knowledge and consist of a name, description, and references.

- Five relationship types between concepts, which create the structure of the ontology. The core relationships are "is sub-concept of," "is pre-requisite," and "is similar to," yet in principle extendable with any relationship. The "is sub-concept of" relationship introduces a hierarchy of concepts.

- Skills associated with concepts, which detail the practical use of EO*GI knowledge.

In previous BoKs, the focus on academic content rather than how it is applied in practice resulted in minimal adoption, especially by practitioners from outside the core of EO* GI and by stakeholders outside of academia (Rickles, Ellul, \& Haklay, 2017). To help address this concern, "learning outcomes" in predecessor BoK versions (see Figure 1), which emphasize the educational perspective, have been replaced by more generically described skills, allowing use for different purposes. For example, it eases the link to tasks as required for occupational profile descriptions and job offer formulations, thus strengthening the business perspective in the BoK. At the same time, the BoK still remains relevant for the education/academic community, as the skills identified can be seen as skills to be acquired and developed through education and training.

To have a consistent description, guidelines for the formulation of skills by domain experts have been defined. Within EO4GEO, a skill needs to be specified using a verb, selected from a revised version of the taxonomy of educational objectives by Anderson and Krathwohl (2001), which itself is based on Bloom, Engelhart, Furst, Hill, and Krathwohl's (1956) taxonomy, and a statement based on a concept of the BoK. The taxonomy from Anderson and Krathwohl (2001) has been complemented with verbs that are relevant for EO*GI-related tasks (e.g., implement, perform, deliver). The taxonomy foresees six levels of skills, ranging from more abstract, lower-level skills (remember and understand) to more concrete, higher-level skills (apply, analyze, evaluate, create) (Figure 2). Examples of skills formulated using the revised taxonomy are:

- "Outline the use of a reference data set in the context of an accuracy assessment"-a skill linked to the BoK concept "accuracy assessment," positioned on level 2 of Bloom's revised taxonomy (understand).

- "Perform an accuracy assessment based on a reference data set"-a skill linked to the BoK concept "accuracy assessment," positioned on level 3 of Bloom's revised taxonomy (apply).

From a business-oriented perspective, for example for use in a job profile, it is generally assumed that a potential employee has relevant background knowledge, and therefore practical skills related to tasks that the workforce needs to perform are more relevant. Such skills are generally located on higher levels of the taxonomy (Anderson \& Krathwohl, 2001). On the contrary, from an educational perspective, the foundations of a domain, 


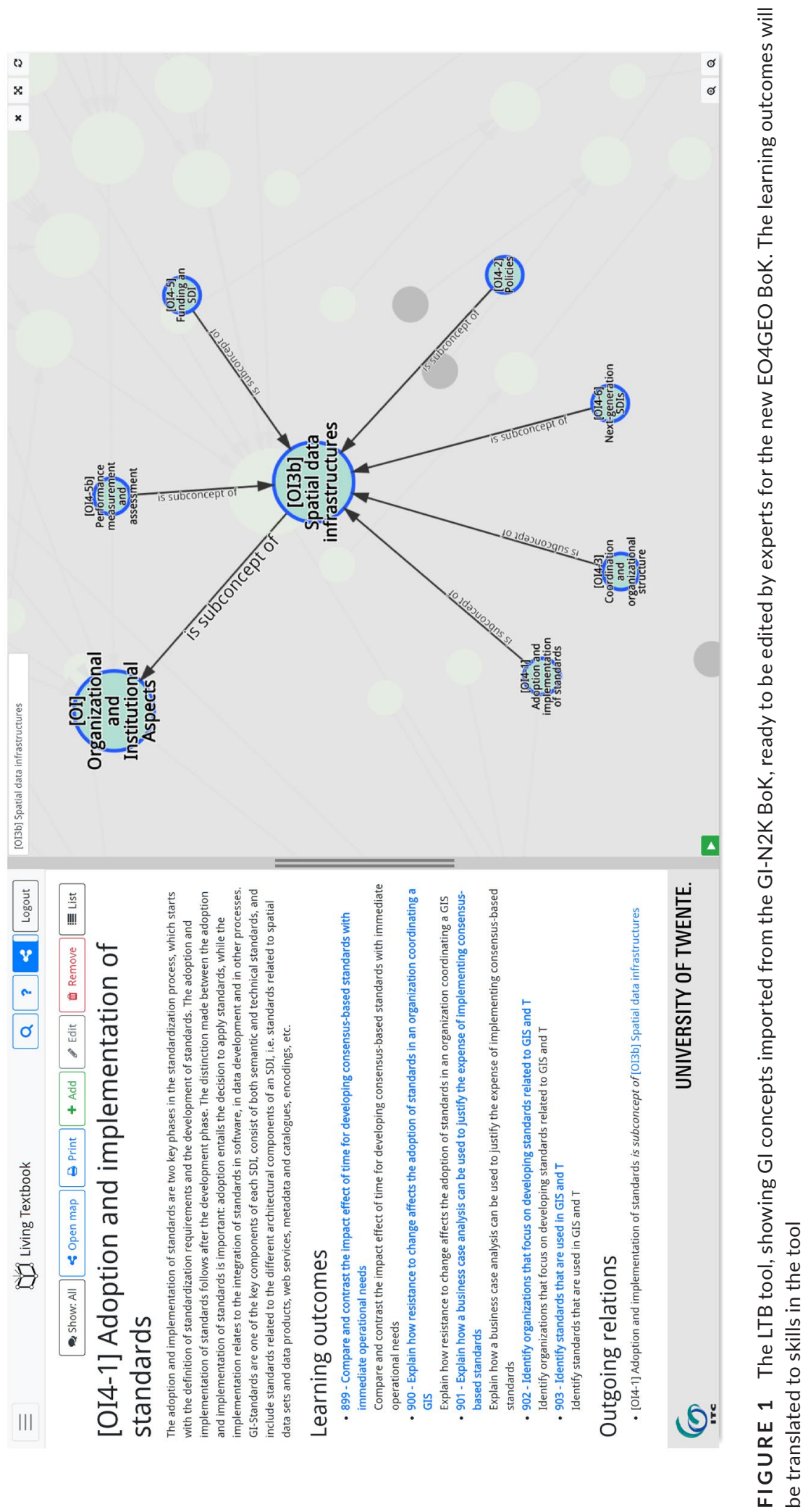




\begin{tabular}{|c|c|c|c|c|c|}
\hline $\begin{array}{c}1 \\
\text { remember }\end{array}$ & $\frac{2}{\text { understand }}$ & $\begin{array}{c}3 \\
\text { apply }\end{array}$ & $\begin{array}{c}4 \\
\text { analyze }\end{array}$ & $\begin{array}{l}5 \\
\text { evaluate }\end{array}$ & $\begin{array}{c}6 \\
\text { create }\end{array}$ \\
\hline $\begin{array}{l}\text { choose } \\
\text { define } \\
\text { find } \\
\text { identify } \\
\text { list } \\
\text { locate } \\
\text { name } \\
\text { recognize } \\
\text { relate } \\
\text { remember } \\
\text { select } \\
\text { state } \\
\text { write }\end{array}$ & $\begin{array}{l}\text { cite } \\
\text { classify } \\
\text { compare } \\
\text { contrast } \\
\text { deliver } \\
\text { demonstrate } \\
\text { discuss } \\
\text { estimate } \\
\text { explain } \\
\text { illustrate } \\
\text { indicate } \\
\text { interpret } \\
\text { outline } \\
\text { relate } \\
\text { report } \\
\text { review } \\
\text { understand }\end{array}$ & $\begin{array}{l}\text { apply } \\
\text { build } \\
\text { calculate } \\
\text { choose } \\
\text { classify } \\
\text { construct } \\
\text { correlate } \\
\text { demonstrate } \\
\text { develop } \\
\text { identify } \\
\text { illustrate } \\
\text { implement } \\
\text { interpret } \\
\text { model } \\
\text { organise } \\
\text { perform } \\
\text { plan } \\
\text { relate } \\
\text { represent } \\
\text { select } \\
\text { solve } \\
\text { teach } \\
\text { use }\end{array}$ & $\begin{array}{l}\text { analyse } \\
\text { arrange } \\
\text { choose } \\
\text { classify } \\
\text { compare } \\
\text { differentiate } \\
\text { distinguish } \\
\text { examine } \\
\text { find } \\
\text { install } \\
\text { list } \\
\text { order } \\
\text { prioritize } \\
\text { query } \\
\text { research } \\
\text { select }\end{array}$ & $\begin{array}{l}\text { assess } \\
\text { check } \\
\text { choose } \\
\text { compare } \\
\text { decide } \\
\text { defend } \\
\text { determine } \\
\text { estimate } \\
\text { evaluate } \\
\text { explain } \\
\text { interpret } \\
\text { judge } \\
\text { justify } \\
\text { measure } \\
\text { prioritize } \\
\text { recommend } \\
\text { select } \\
\text { test } \\
\text { validate }\end{array}$ & $\begin{array}{l}\text { add to } \\
\text { build } \\
\text { change } \\
\text { choose } \\
\text { combine } \\
\text { compile } \\
\text { construct } \\
\text { convert } \\
\text { create } \\
\text { design } \\
\text { develop } \\
\text { devise } \\
\text { discuss } \\
\text { estimate } \\
\text { manage } \\
\text { model } \\
\text { modify } \\
\text { plan } \\
\text { process } \\
\text { produce } \\
\text { propose } \\
\text { revise } \\
\text { solve } \\
\text { test } \\
\text { transform }\end{array}$ \\
\hline
\end{tabular}

FIGURE 2 The verb taxonomy used to formulate skills in the EO4GEO BoK (adapted from Anderson \& Krathwohl, 2001)

theories, and background knowledge are more relevant for describing course objectives, and thus related skills are generally linked to the lower levels of Anderson's taxonomy.

To create the concepts, relationships between them, and skills that altogether constitute the ontology-based EO4GEO BoK, the living textbook (LTB) environment is used (Lemmens, Ronzhin, Augustijn, Verkroost, \& Walsh, 2018). LTB is a web tool developed by the University of Twente for modeling and visualizing domain knowledge for the purpose of education and knowledge exchange. Its interface combines a wiki-style text window and a concept map (Figure 3). With this tool, domain experts and teachers, in their role as content developers, can collaboratively create descriptions of concepts and connect them via self-defined relationships. The granularity of the concepts is chosen by the content developers, normally based on the perception of what are the domain's core building blocks. With relationships such as "is a kind of," a hierarchy can be created and links like "is a part of," "makes use of," etc. can be utilized to enrich specific concept semantics. The tool builds upon the principles of the Semantic Web and Linked Open Data. It offers the visualization of learning paths and learning analytics tools for deployment in education and has built-in functions to manage user roles for content viewers, editors, reviewers, and administrators.

Domain experts can define new concepts and change existing ones, connect them and add skills, external resources, etc., altogether forming the EO4GEO BoK. The BoK content can be exported for external use (e.g., to feed tools supporting curriculum development, creating job descriptions, and profiles of the EO workforce).

At present, the BoK content is accessible for researchers, $\mathrm{EO} * \mathrm{Gl}$ specialists who have registered in the EO4GEO network of experts (http://www.eo4geo.eu/eo4geo-call-for-experts/). This network is currently contributing to the extension of the BoK for $\mathrm{EO}^{*} \mathrm{Gl}$ by updating and identifying new concepts, including the formulation of skills. The EO4GEO BoK will be made available openly in the foreseeable future. 


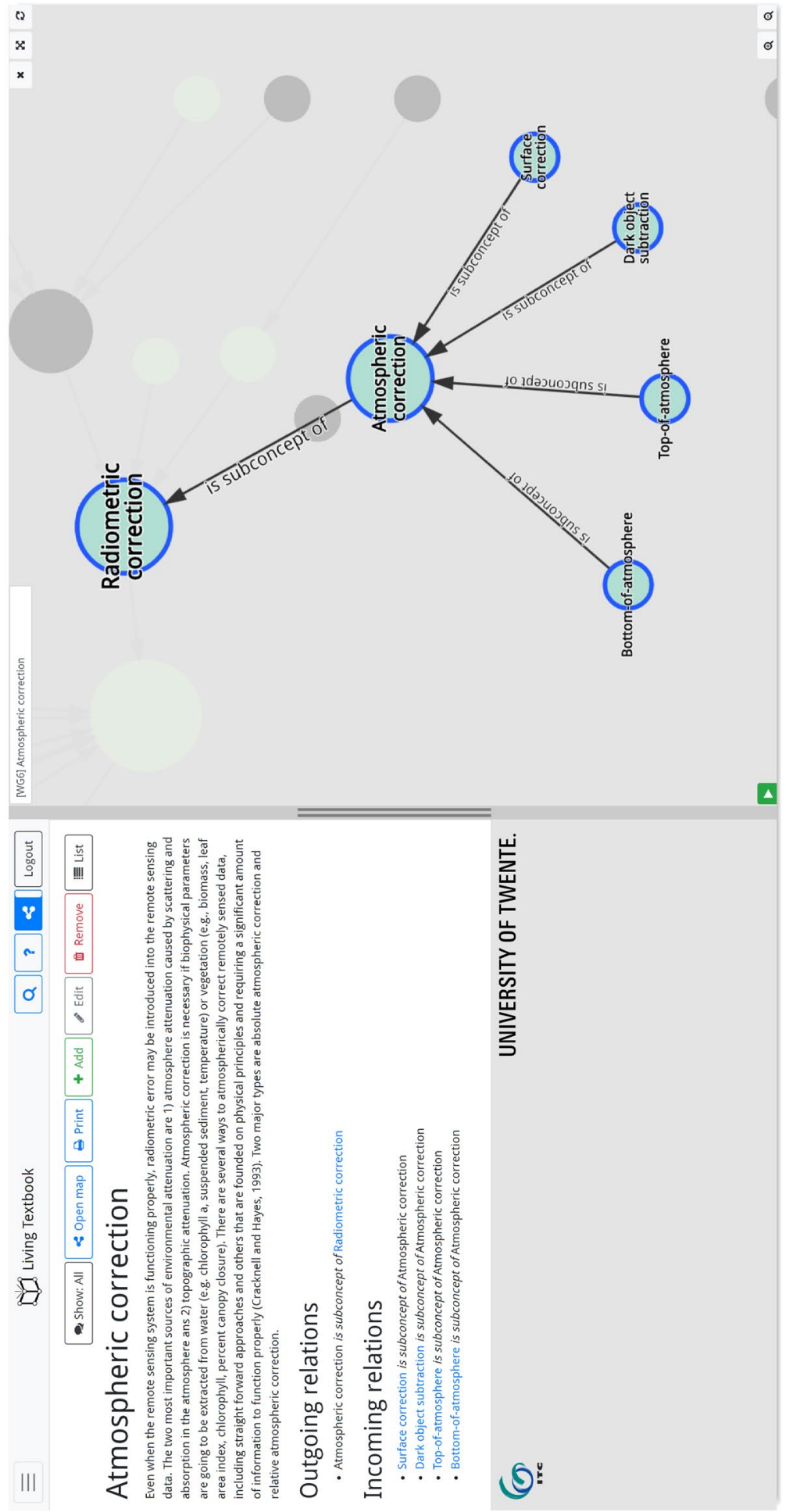

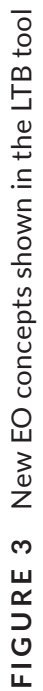




\section{4 | BUSINESS PROCESS DESCRIPTIONS AS A MEANS TO DOCUMENT THE SKILL REQUIREMENTS OF A WORKFORCE}

Next to the transition from learning outcomes to skills, the expert-based revision process is complemented with an approach to analyze business processes, specified by professionals in the EO* Gl field, to derive practical tasks, skills, and related BoK concepts. This further strengthens the industry perspective of the BoK, assures agreement on the wording of the BoK content acceptable for industry and academia, and may eventually lead to curricula corresponding to market needs. Business processes as a series of coordinated tasks are performed in order to achieve a defined set of results. To illustrate business process flows, the business process model and notation (BPMN) provides a standardized way of representation (OMG, 2014). BPMN can contain multiple levels of process details, differentiable through sub-processes and tasks. A strength of business process models is the indication of stakeholders involved in the work and stakeholders' views with regard to how the process applies to them (Rosing, Scheer, \& Scheel, 2015).

In the course of the EO4GEO project, 30 business processes were collected that cover various workflows currently performed in companies and research institutions active in the $\mathrm{EO}{ }^{*} \mathrm{GI}$ domain. The provided workflows list the main tasks and stakeholders, which in turn give an indication of the skills needed and the concepts involved. More specifically, tasks are defined as skills and knowledge applied in a specific application context (cf. Cedefop, 2014). This business process analysis allows us to identify occupational profiles in the workforce directly associated with the EO*GI domain, as well as profiles of the workforce of neighboring domains that interact with representatives of the EO* GI domain. Furthermore, it allows the evaluation of BoK content (i.e., concepts and skills), as contrasting the results of the business process analysis with existing BoK content leads to confirming or updating it, or extending the BoK with new content.

An example of a business process in the EO* GI domain is displayed in Figure 4. The involved stakeholders are a geological authority, a GIS expert, and an EO analyst who complete different tasks in a workflow for updating a

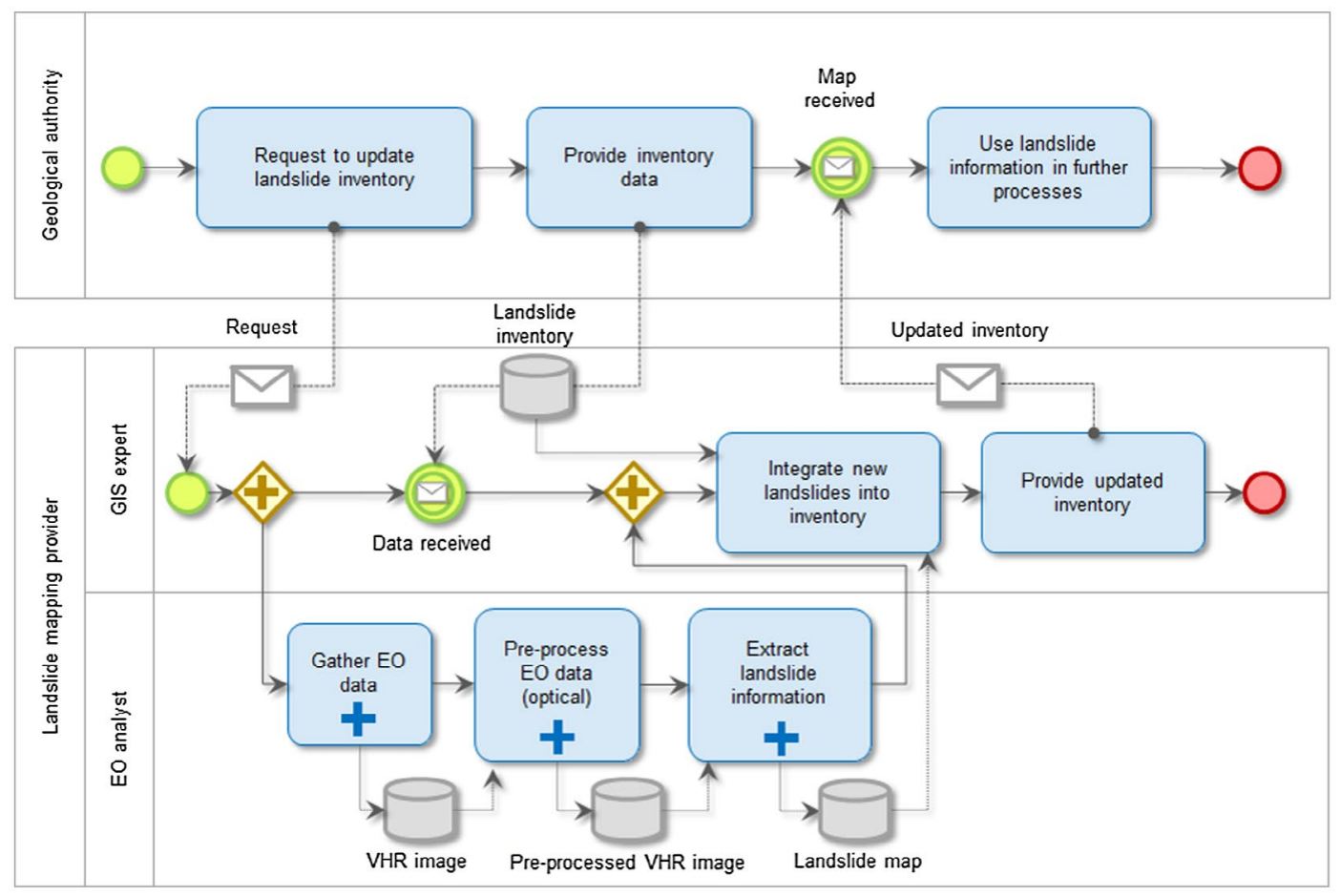

FIGURE 4 Exemplary business process describing the interaction between stakeholders 
landslide inventory. The plus sign next to the tasks of the EO analyst indicates that these boxes are further specified in sub-processes.

The analysis of the business processes enabled the identification of more than 200 tasks that constitute a rich source of information on which tasks the EO*GI workforce needs to be able to perform. If the EO4GEO BoK is claimed to be useful from an industry perspective, then the concepts and skills providing the foundation for performing these tasks need to be contained in the EO4GEO BoK. Only if this requirement is fulfilled will the BoK constitute a vocabulary for describing tasks from an industry perspective as well as learning outcomes of curricula.

The detailing of tasks based on BoK content is an interactive process between experts involved in the tasks of a business process and experts knowing the details of concepts included in the BoK. The following sections demonstrate an example of a mapping of tasks to skills and concepts, and the lessons learnt from an exercise involving a larger group of people performed in a workshop.

\section{5 | MAPPING BETWEEN TASKS, SKILLS, AND CONCEPTS}

The idea behind the approach of mapping tasks to skills and concepts is illustrated with a metaphor: concepts and skills as included in the BoK form the fertile ground on which plants-representing tasks-can grow (Figure 5). The description of tasks can require various combinations of skills and concepts, however, there is a finite number of skills and concepts required for describing tasks. The mapping serves the purpose of detailing what tasks are involved based on the content of the BoK.

This approach requires experts' input to break down large tasks into sub-tasks, and then to identify the concepts and skills related to the sub-tasks. For example, it might be obvious for experts what the task "perform land cover classification" entails, even though this task can be sliced into a series of further components. The decomposition and detailed specification of sub-tasks of the overarching tasks is essential to cover practical workforce tasks, when designing focused learning modules aimed to cover practical needs (e.g., vocational training), and when defining broader curricula and training programs that need to cover the concepts and skills required to perform a particular higher-level task, such as "perform land cover classification." A task like "apply geometric correction," in contrast, is much more specific and might not require further specification.
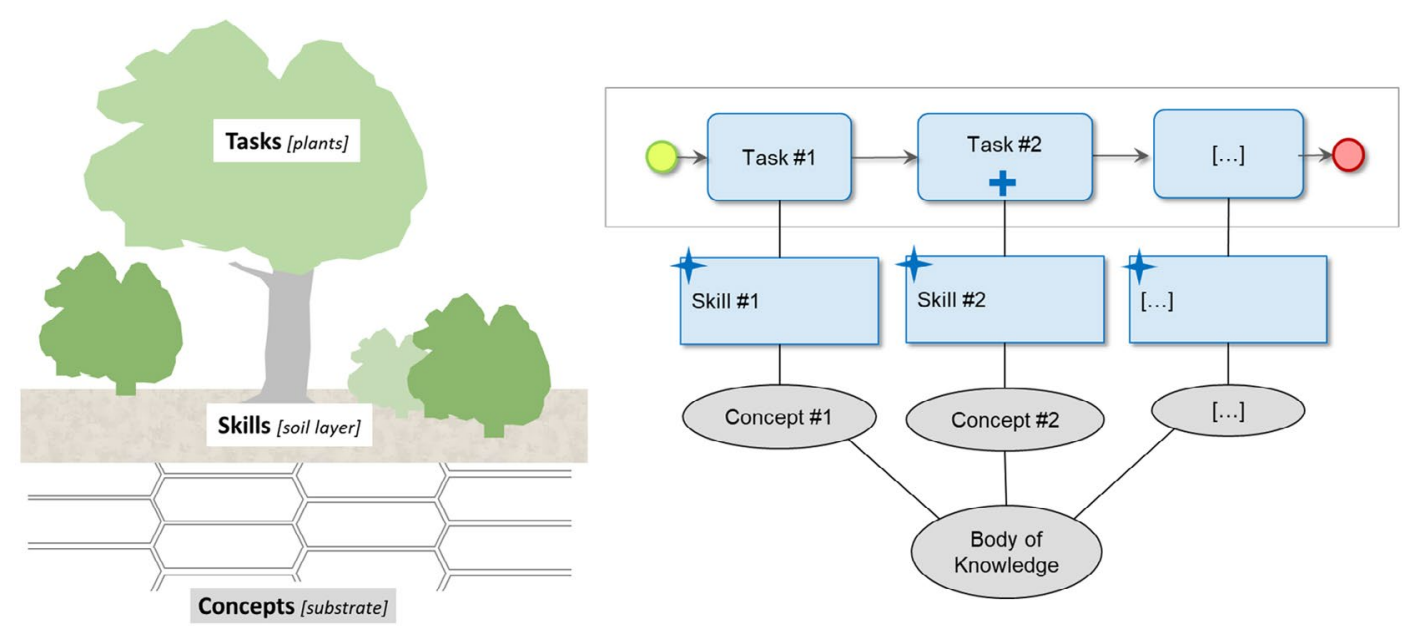

FIGURE 5 (a) Metaphor demonstrating the interplay between tasks, skills, and concepts as included in the BoK; and (b) representation of the metaphor in a mapping diagram 


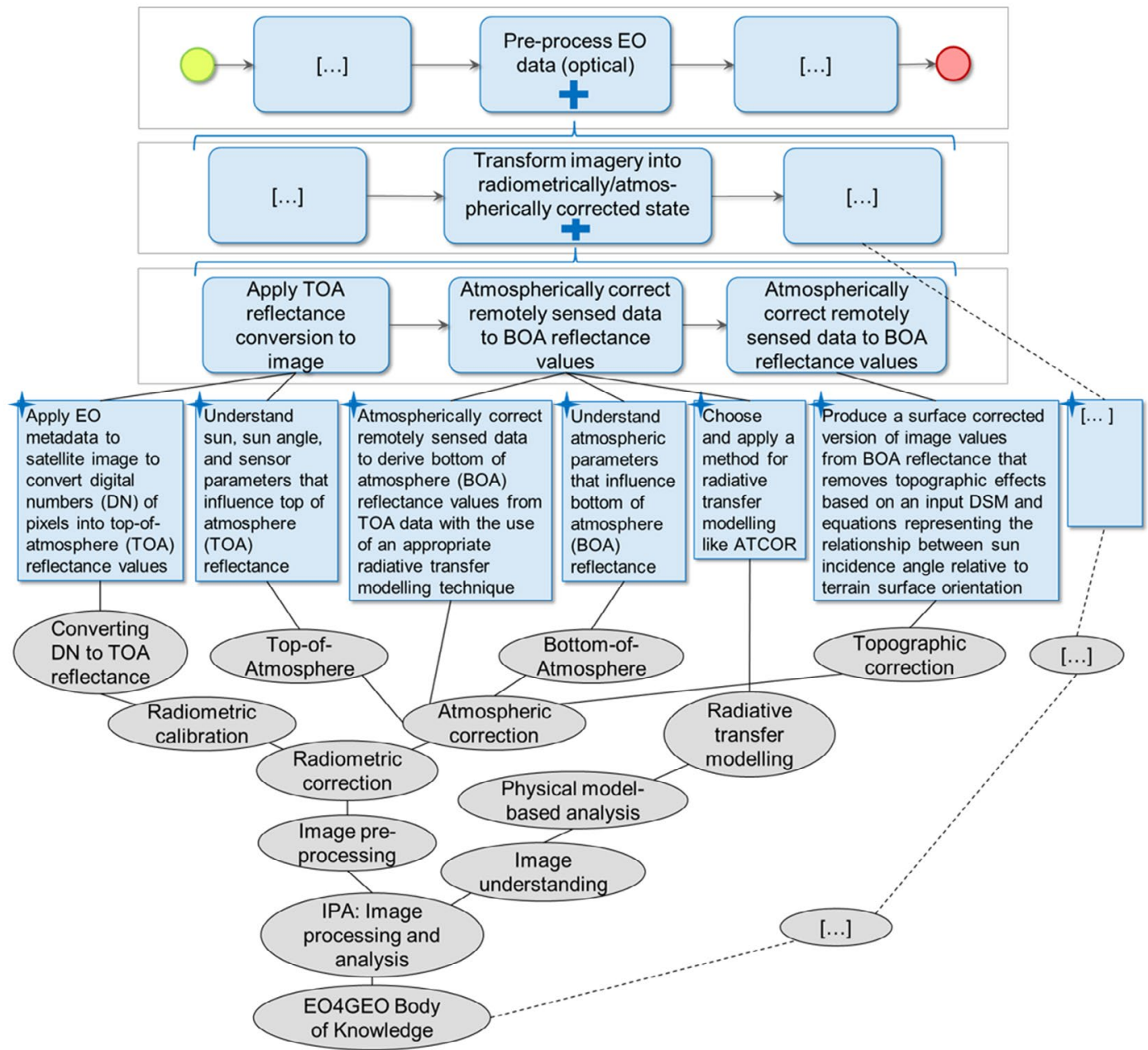

FIGURE 6 Mapping of the task "pre-process EO data (optical)" to skills and concepts of the EO4GEO BoK; blue boxes are tasks and sub-tasks derived from a business process model (cf. Figure 4); blue boxes with stars are skills and gray ellipses are concepts of the EO4GEO BoK

Figure 6 contains an example for the mapping of a part of the business process specified in Figure 4, namely the task "pre-process EO data (optical)" is decomposed into its sub-tasks and linked to related skills and concepts. To achieve this mapping, the BoK has then been searched for concepts that represent knowledge related to the task at hand, which led to the indication of BoK concepts linked to skills, or the identification of missing BoK content, which in turn feeds the BoK revision process. The concepts linked to skills in the provided example are all sub-concepts of the "image processing and analysis" concept, yet the link with skills has been introduced in the most detailed concept related to a skill. The relations between the concepts are defined by "is sub-concept of" relationships in the BoK ontology.

The use of skills in this example indicates the close relation between sub-tasks and skills. In fact, the skills shown in Figure 6 have been introduced during the mapping process, as the current EO4GEO BoK does not yet contain detailed concept descriptions and skills associated with concepts for the newly introduced EO concepts. The skills in the example follow the guidelines introduced in the previous section, of formulating skills as verb plus concept-based statement, taking the revised taxonomy of Anderson and Krathwohl (2001) into consideration.

Figure 7 shows an additional example of the usefulness of tasks for the formulation of skills. It contains a mapping between tasks and concepts, where the tasks indicate skills related to the concept "accuracy assessment." 


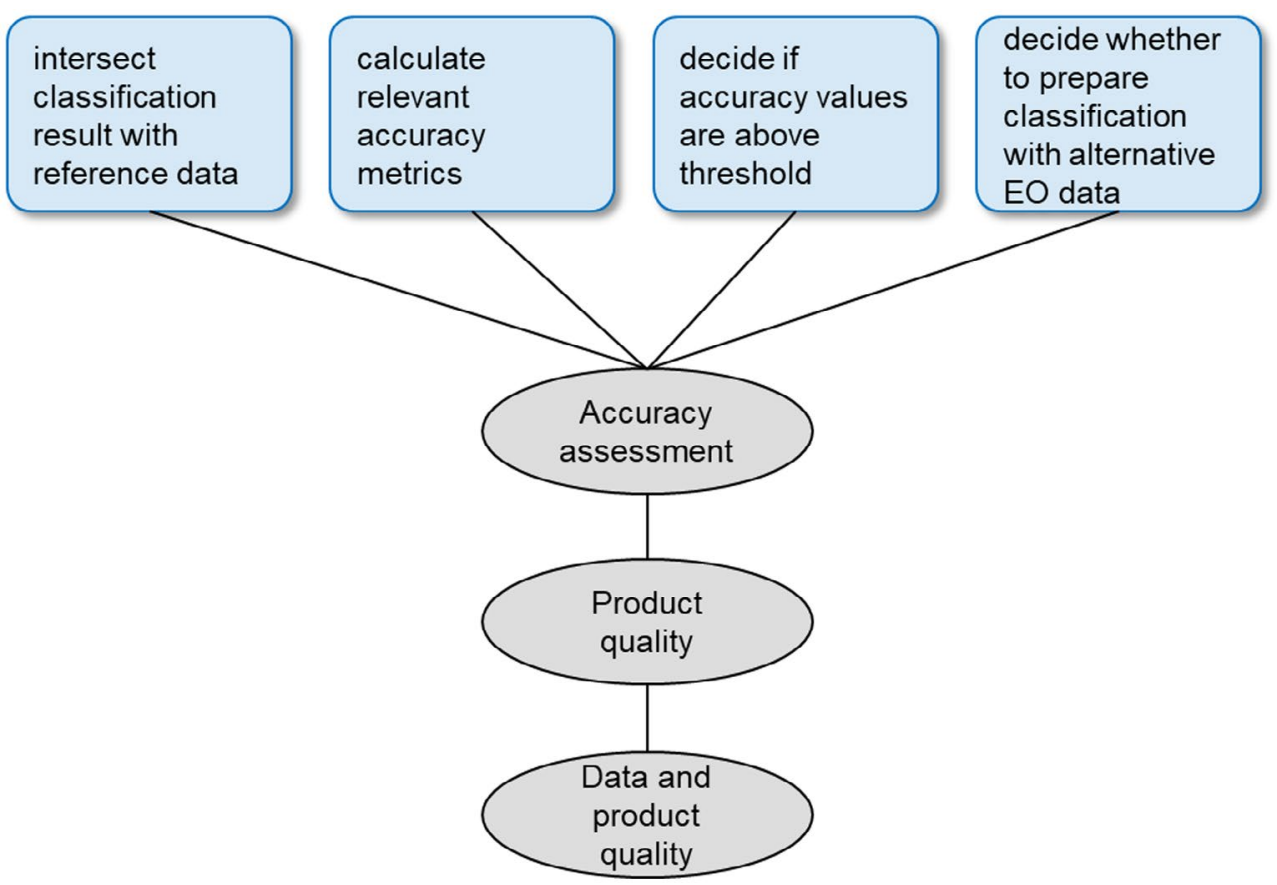

FIGURE 7 Tasks related to the concept "accuracy assessment" that serve as input for the formulation of skills

The more precisely tasks are formulated and the closer their formulation follows the proposed formulation of skills (verb plus concept-based statement), the better their applicability for the formulation of skills.

\section{6 | EXPERIENCES FROM A WORKSHOP AND DISCUSSION OF THE APPROACH}

As the mapping between tasks, concepts, and skills is a means to analyze the details of tasks of the EO* GI workforce, a workshop has been organized to leverage the expertise of those people who had previously contributed business process descriptions. The workshop took place in Warsaw in November 2019 and involved around 40 people, most of them EO4GEO project partners, both from academia and industry.

The materials presented to facilitate the work in the mapping exercise were: the list of identified tasks, access to the currently available concepts of the EO4GEO BoK in the LTB tool, examples of mapping from tasks to concepts and skills. It needs to be noted that the description of concepts in the EO4GEO BoK is still ongoing, and in many cases associated skills are still missing. In addition, it was the first encounter with the EO4GEO BoK implemented in the LTB tool for the workshop participants, and also their first encounter with the large collection of EO concepts that have been added to the EO4GEO BoK.

The exchange with workshop participants proved useful for the evaluation of the current state of the EO4GEO BoK. Workshop participants gained experience in the content of the BoK, its structure, and its implementation in the LTB tool. This practical insight of the BoK proved useful for increasing the understanding of what the BoK can offer its users. In some instances, concepts were identified that were missing in the current version, as for example the concept covering "cube sats," or concepts at detailed levels of the hierarchy required for mapping tasks. In addition, a discussion on the inclusion of markets for the EO sector started within EO4GEO as a consequence of the direct interaction with the BoK. 

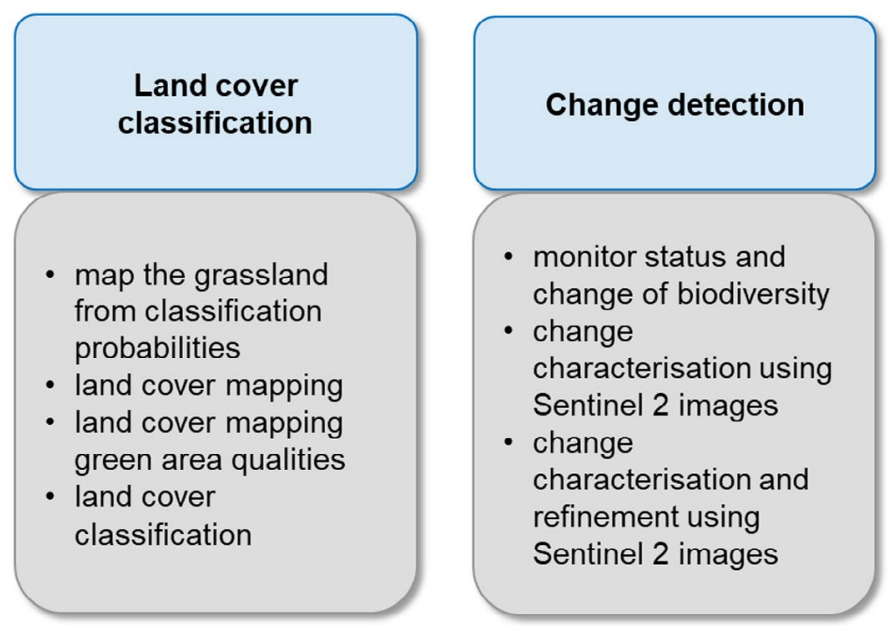

FIGURE 8 Examples of different formulations used in business process descriptions that refer to the same two tasks

The LTB tool proved to be a valuable tool for exploring and understanding the meaning of BoK concepts and their context, as it represents them as a combination of text descriptions and a visualization of the ontology. The use of the LTB tool led to important feedback on its further development. Crucial for the exploration of the BoK is the search functionality in the tool, given the expected large number of concepts. As the current EO4GEO BoK lacks synonyms for concepts, users of the tool have to search with alternative keywords for finding the appropriate concept. These difficulties were overcome quickly after a short period of time of interacting with the LTB tool.

The success of the mapping of tasks to concept and skills largely depends on the concise formulation of tasks when describing business processes. So, in case the quality of business process descriptions does not fit the needs of the intended analysis and translation to concepts and skills, a revision of the provided task descriptions might be advisable (e.g., by asking to elaborate tasks further into sub-tasks, to achieve an appropriate level of detail). Examples of tasks that cover a series of implicit or explicit sub-tasks and need to be broken down into these subtasks before a mapping can be applied are:

- "process satellite data (convert to reflectance and crop to AOI) and store in local EO storage";

- "delineate burned area using Sentinel-2 images";

- "product integration and validation";

- "information products and metadata preparation";

- "produce map with maximum surface temperatures."

Tasks are generally linked to a specific application context and need to be abstracted from this application context to be mapped to concepts in the BoK. Examples of tasks that require abstraction are:

- "add population information";

- "correlate with weather station information (air temperature vs. surface temperature)";

- "delineation of natural areas that are important for infiltration and delaying of the water";

- "SPW provides new aerial images to ISSeP."

The mapping exercise highlighted inconsistent formulations used in task descriptions. Figure 8 exemplifies this observation for two sets of tasks that can be summarized into the tasks "land cover classification" or "change 
detection," respectively. This demonstrates the potential of the task analysis approach to homogenize domain terminology across business model descriptions, and mediate on a shared vocabulary of the domain, captured in the BoK.

The translation of tasks into skills and concepts was not trivial for the workshop participants. On the one hand, the experts on the tasks had to verbalize their tacit knowledge about the tasks and on the other hand, they had to be aware of the BoK content. The fact that the EO4GEO BoK is not available in its final version, with full concept descriptions and skills associated with the concepts, and the fact that the tool used was new certainly increased the difficulty of the tasks for the participants. It also turned out that the granularity of task descriptions, as well as imprecise or business-specific terminology used in task naming, constitute a challenge in mapping tasks to concepts and skills of the BoK. The examination of concepts and skills involved in tasks is a positive feedback loop to specify the terminology used for tasks and the level of detail of task descriptions.

There are currently two parallel efforts to automatically translate tasks into skills and concepts. One is the Open Knowledge Network for Spatial Decision Support technologies, where partners from the New Jersey Institute of Technology and University of Arizona are working to parse the meaning in tens of thousands of journal articles to create knowledge graphs depicting the concepts underlying those articles (as they relate to spatial decision support). Our own efforts skim closer to the task orientation that underlies the EO4GEO BoK, where we catalog all verbs and derive the semantic contexts within which they are employed. Those phrases are then subjected to a weakly supervised hierarchical text classification (Meng, Shen, Zhang, \& Han, 2019) to determine the position of the task within the larger EO4GEO BoK.

The mapping approach was demonstrated to be useful for evaluating the status quo of the EO4GEO BoK and for initiating a discussion with professionals in the field on what tasks comprise. Using the tasks collected from business process descriptions as a foundation for this exercise has shortcomings, because the business processes reflect the current state-of-the-art of work in the $\mathrm{EO}^{*} \mathrm{Gl}$ domain and provide little indication of cutting-edge approaches used. This approach is therefore no replacement for a BoK revision process involving domain experts (e.g., interviews, workshops), who provide feedback on the BoK content from the frontiers of technological and scientific developments.

\section{7 | CONCLUSIONS AND FUTURE WORK}

The approach presented in this article emphasizes the use of tasks from business processes for detailing and evaluating the content of the EO4GEO BoK from a business-oriented perspective. Overall, the BoK proved useful by providing a vocabulary of concepts for describing tasks and sub-tasks in an unambiguous manner. However, the experiences from a workshop where the approach was applied show that the integration of a business perspective into the EO4GEO BoK requires repeated interaction between professionals working on these tasks and experts knowing the content of the EO4GEO BoK. Both parties need to work in symbiosis and agree on the vocabulary of the domain that is documented in the BoK. The process of specifying the vocabulary gets more challenging when including people from outside the $\mathrm{EO}^{*} \mathrm{GI}$ domain, as these people have difficulty following the specific terminology of the domain (Rickles et al., 2017).

As the tasks used in this work originate from business processes, they represent the state-of-the-art of work in the industry. The detailed analysis of tasks and sub-tasks indicates which concepts of the BoK are frequently required for completing tasks, thus constituting core BoK content from an industry perspective. In addition, it also allows us to identify gaps in the BoK, namely when appropriate lower-level concepts cannot be identified to describe tasks. Therefore, the approach of decomposing business processes and mapping their tasks to BoK concepts and skills is a means to evaluate the BoK from a business perspective and identify missing concepts and skills to complement its academic-oriented content. 
The rapid technological advances in the sector will strongly affect the requirements for future occupational profiles. Although the sector has emerged from digitalization processes starting $40+$ years ago, the current disruptive changes triggered by the new big (Earth) data paradigm in the context of artificial intelligence and automation in general needs to be reflected in a dynamic BoK. A strong interaction between academic and professional education providers and industry is required, not only to jointly specify a harmonized vocabulary, but also in terms of application fields, and the required tasks and related skills.

The tasks collected through business process descriptions are available to experts involved in the revision of the EO4GEO BoK and they are asked to consider them when adding descriptions of concepts and skills. Also, additional workshops with domain experts are planned to evaluate the BoK content, where the mapping exercise could be repeated.

\section{ORCID}

Barbara Hofer (iD https://orcid.org/0000-0001-7078-3766

\section{REFERENCES}

Abran, A., Moore, J. W., Bourque, P., Dupuis, R., \& Tripp, L. (2004). Software engineering body of knowledge. Washington, DC: IEEE.

Ahearn, S. C., Icke, I., Datta, R., DeMers, M. N., Plewe, B., \& Skupin, A. (2013). Reengineering the GIS\&T body of knowledge. International Journal of Geographical Information Science, 27(11), 2227-2245.

Anderson, L. W., \& Krathwohl, D. R. (2001). A taxonomy for learning, teaching and assessing: A revision of Bloom's taxonomy of educational objectives. New York, NY: Longman.

Bloom, B. S., Engelhart, M. D., Furst, E. J., Hill, W. H., \& Krathwohl, D. R. (1956). Taxonomy of educational objectives: The classification of educational goals (Handbook 1, Cognitive Domain). New York, NY: Longman.

Cedefop. (2014). Terminology of European education and training policy: A selection of 130 terms. Retrieved from https:// europass.cedefop.europa.eu/education-and-training-glossary/

DiBiase, D., DeMers, M., Johnson, A., Kemp, K., Luck, A. T., Plewe, B., \& Wentz, E. (2007). Introducing the first edition of geographic information science and technology body of knowledge. Cartography and Geographic Information Science, 34(2), 113-120.

Frazier, A. E., Wikle, T., \& Kedron, P. (2017). Exploring the anatomy of Geographic Information Systems and Technology (GIS\&T) textbooks. Transactions in GIS, 22(1), 165-182.

Gruijthuijsen, W., Pontes, S., Vancauwenberghe, G., Vandenbroucke, D., \& Olijslagers, M. (2019). Understanding skill needs in the EO/GI sector: A job advertisements analysis. In P. Kyriakidis, D. Hadjimitsis, D. Skarlatos, \& A. Mansourian (Eds.), Accepted short papers and posters from the 22nd AGILE Conference on Geo-information Science, Limassol, Cyprus. Retrieved from https://agile-online.org/programme-2019/accepted-papers-and-posters-2019

Kedron, P., Frazier, A., Greene, C., \& Mitchell, D. (2016). Curriculum design for upper and advanced-level GIS classes: Are new skills being taught and integrated? Journal for Geographic Information Science, 4(1), 324-335.

Lemmens, R. L. G., Ronzhin, S., Augustijn, P. W. M., Verkroost, M. J., \& Walsh, N. (2018). Space education with the living textbook, a web-based tool using a concept browser. In Proceedings of the Second Symposium on Space Educational Activities, Budapest, Hungary.

Meng, Y., Shen, J., Zhang, C., \& Han, J. (2019). Weakly-supervised hierarchical text classification. In Proceedings of the 33rd AAAI Conference on Artificial Intelligence, Honolulu, HI (pp. 6826-6833). Menlo Park, CA: AAAI.

Morris, P. W. G., Patel, M. B., \& Wearne, S. H. (2000). Research into revising the APM project management body of knowledge. International Journal of Project Management, 18(3), 155-164.

Mundie, D. A., \& Ruefle, R. (2012). Building an incident management body of knowledge. In Proceedings of the Seventh International Conference on Availability, Reliability and Security, Prague, Czech Republic (pp. 507-513). Piscataway, NJ: IEEE.

OMG. (2014). Business process model and notation (BPMN), version 2.0.2. Retrieved from http://www.omg.org/spec/ BPMN

Rickles, P., Ellul, C., \& Haklay, M. (2017). A suggested framework and guidelines for learning GIS in interdisciplinary research. Geo: Geography \& Environment, 4(2), 1-18.

Toppen, F., \& Reinhardt, W. (2009). A European approach towards the UCGIS Geographic Information Science and Technology Body of Knowledge: A discussion paper. In Proceedings of the 12th Annual AGILE International Conference on Geographic Information Science, Hannover, Germany. 
Vandenbroucke, D., \& Vancauwenberghe, G. (2016). Towards a new body of knowledge for Geographic Information Science and Technology. International Scientific Journal: Micro Macro \& Mezzo Geo Information, 6, 7-19.

vonRosing, M., Scheer, A. W., \& vonScheel, H. (2015). The complete business process handbook: Body of knowledge from process modeling to BPM (Vol. I). Waltham, MA: Morgan Kaufmann.

Wallentin, G., Hofer, B., \& Traun, C. (2015). Assessment of workforce demands to shape GIS\&T education. Transactions in GIS, 19(3), 439-454.

Wilson, J. P. (2014). Geographic Information Science \& Technology: Body of Knowledge 2.0 Project (Final report). Retrieved from https://ucgis.memberclicks.net/assets/docs/ucgis_bok2_wilson_report_dec2014.pdf

How to cite this article: Hofer B, Casteleyn S, Aguilar-Moreno E, et al. Complementing the European earth observation and geographic information body of knowledge with a business-oriented perspective.

Transactions in GIS. 2020;24:587-601. https://doi.org/10.1111/tgis.12628 